\title{
Prevalence of foveolar lucency with different gas tamponades in surgically closed macular holes assessed by spectral domain optical coherence tomography
}

\author{
Javier Zarranz-Ventura, MD, PhD, FEBO, ${ }^{1,2,5}$ Abdallah A. Ellabban, MD, , 1,3 \\ Dawn A. Sim, PhD, FRCOphth, ${ }^{5}$ Pearse A. Keane, MD, FRCOphth, ${ }^{5}$ James \\ N. Kirkpatrick, MD, FRCOphth, ${ }^{1}$ Ahmed A. B. Sallam, PhD, FRCOphth ${ }^{1,4}$ \\ ${ }^{1}$ Vitreo-Retinal Service, Cheltenham General Hospital, Gloucestershire Hospitals NHS Trust, \\ Cheltenham, United Kingdom \\ ${ }^{2}$ Vitreo-Retinal Unit, Institut Clínic d'Oftalmologia (ICOF), Hospital Clínic, Barcelona, Spain \\ ${ }^{3}$ Department of Ophthalmology, Suez Canal University, Ismailia, Egypt \\ ${ }^{4}$ Jones Eye Institute, University of Arkansas for Medical Science, Arkansas, USA \\ ${ }^{5}$ NIHR Biomedical Research Centre for Ophthalmology, Moorfields Eye Hospital NHS \\ Foundation Trust, London, United Kingdom
}

\section{Correspondence and reprint requests:}

Javier Zarranz-Ventura, Vitreo-Retinal Service, Cheltenham General Hospital, Sandford Road, Gloucestershire Hospitals NHS Trust, Cheltenham, United Kingdom (jzarranz@hotmail.com).

Tel: +34660008417

\section{Acknowledgements:}

This work is dedicated to the memory of Rob Johnston, Vitreo-Retinal Consultant (1966-2016), who played a major role in designing this study and passed away weeks before the submission of this paper. Rest in peace Rob. 


\section{Disclosure:}

None of the authors have any conflict or proprietary interest in any of the devices mentioned in this article.

\section{Running Head:}

Foveolar lucency and gas tamponade

\section{Keywords:}

Ellipsoid

Foveolar lucency

Foveal lucency

Gas

Macular hole

Neurosensorial

Optical coherence tomography

Subretinal fluid

Tamponade

Vitrectomy

\section{Summary statement:}

Temporary foveolar lucency is a highly prevalent feature in successfully closed macular holes at month 1 , which seems to be related to the gas tamponade type employed and does not affect the final visual outcome. 


\section{Abstract:}

Purpose: To evaluate the prevalence of foveolar lucency (FL) in surgically closed macular holes by spectral domain optical coherence tomography (SDOCT).

Methods: 142 eyes of 132 patients underwent pars plana vitrectomy (PPV), internal limiting membrane (ILM) peeling and gas tamponade in a 60 months' time frame. Anatomical success and FL rates assessed by SD-OCT, mean preoperative and postoperative best-measured visual acuity (BMVA) and surgical details were retrospectively analyzed.

Results: SD-OCT confirmed closed holes with FL in 33.7\% (34/101) of eyes at 1 month, $7.3 \%(9 / 123)$ at 3 months, $4.6 \%(6 / 129)$ at 6 months and $3 \%$ (4/133) at 12 months. Prevalence of FL in closed holes at month 1 was lower in C3F8-treated eyes $(9.5 \%, 2 / 21)$ compared to C2F6 $(40.9 \%, 18 / 44, p=$ $0.03)$ and SF6-treated eyes $(38.9 \%, 14 / 36, p=0.05)$. No differences were observed at month 3. No differences in BMVA change were observed between closed holes with or without FL at month $1(-0.14 \pm 0.19$ vs. $-0.11 \pm$ $0.23, p=0.48$ ) or any of the other time points.

Conclusions: Temporary FL is a highly prevalent feature in successfully closed macular holes. Eyes treated with C3F8 gas had lower rates of FL at month 1 than C2F6 and SF6-treated eyes. The presence of FL in closed holes does not appear to have any effect on the visual outcomes. 


\section{INTRODUCTION:}

Idiopathic full-thickness macular hole (IFMH) is characterized by a full thickness defect in the fovea that causes a central scotoma, metamorphopsia and reduced visual acuity. ${ }^{1}$ It represents a common vitreo-retinal pathology with an estimated incidence of 8.6 cases $/ 100,000$ population/year ${ }^{2}$ and is the indication for surgery in almost $10 \%$ of all pars plana vitrectomy (PPV) operations performed in the United Kingdom (UK). ${ }^{3}$ The surgical treatment of IFMH with PPV combined with intravitreal gas tamponade has been proven effective in randomized controlled trials for stage II, III and IV holes,,$^{4,5}$ resulting in good long-term visual and anatomical outcomes. ${ }^{6}$ More recently, the results of a prospective registry-based study from New Zealand and Australia demonstrated that peeling of the internal limiting membrane (ILM) increased the anatomical closure rate ${ }^{7}$, although this was not always associated with better visual acuity outcomes. ${ }^{8}$

The use of optical coherence tomography (OCT) has refined the clinical description of success (open or closed) to include analysis of the status of the outer retinal layers in ophthalmoscopically closed holes. ${ }^{9,} 10$ In particular the presence of foveolar lucency $(\mathrm{FL})$ - a hyporeflective space underlying the fovea with continuity of the inner retinal layers after surgery - and/or disruption of outer retinal layers such as the ellipsoid layer or the external limiting membrane have been described in the course of the macular hole closure healing process. ${ }^{9-12}$ Given the descriptive nature of these studies in small cohorts of patients, the prevalence of these findings remains uncertain. 
The primary aim of the study was to determine the prevalence of FL in eyes with closed macular holes after vitrectomy surgery at different time points in a large series of patients. Secondary aims were to explore the influence of the type of intraocular gas tamponade on this OCT feature and its effect on postoperative visual acuity at different time points.

\section{METHODS:}

\section{Study Eyes}

This study included 142 eyes from 132 patients with idiopathic macular hole operated in the vitreo-retinal unit of Gloucestershire Hospitals NHS Foundation Trust by 3 consultant teams and followed up with spectral domain optical coherence tomography (SD-OCT) over a 60 months' period (20072012). All clinical data for these patients were prospectively entered during routine clinical practice into an electronic medical record (EMR) system (Medisoft Ophthalmology, Medisoft Limited, Leeds, UK). Data were extracted and anonymized as part of a non-interventional retrospective audit and on this basis formal ethics approval was not required. All patients underwent 20 or 23G pars plana vitrectomy (PPV), ILM peel and gas tamponade. In all cases ILM peeling was performed with trypan blue and brilliant blue (TB-BB) (Membraneblue-Dual, DORC, Zuidland, The Netherlands). The gas tamponade employed was determined by consultant choice rather than macular hole grade with one consultant favouring sulfahexafluoride (SF6), another favouring hexafluoroethane (C2F6) and a third perfluoropropane (C3F8). All cases were given the same posturing advice: to avoid lying supine 
for the first week regardless of macular hole stage or gas tamponade employed, patients were not instructed to adopt face down positioning. Macular holes secondary to previous retinal surgery, uveitis, diabetes or trauma were excluded from this study.

\section{Clinical Data}

Clinical and demographic data extracted from the EMR included: age, gender, stage of macular hole, type of tamponade, preoperative phakic status, secondary cataract surgery rate, anatomical success rate and FL prevalence by SD-OCT images at 1, 3, 6, 12 and 24 months post-surgery, preoperative and postoperative best measured visual acuity (BMVA) at 1, 3, 6, 12 and 24 months after surgery. Visual acuity was measured in either logarithm minimum angle of resolution (LogMAR) or Snellen notation and converted to LogMAR for analyses.

\section{SD-OCT Image Acquisition Protocol}

All SD-OCT image sets included in this study were acquired on the Cirrus HDOCT (Carl Zeiss Meditec, Dublin, California) during routine clinical practice using the Macular Cube protocol. The Macular Cube protocol consists of 128 horizontally oriented B-scans acquired in a continuous, automated sequence and covers a $6 \mathrm{~mm} \times 6 \mathrm{~mm}$ area. Each B scan is $6 \mathrm{~mm}$ in length and composed of 512 equally spaced transverse sampled locations.

\section{SD-OCT Qualitative and Quantitative Analysis Criteria:}


SD-OCT images were analyzed for macular hole closure postoperatively at different time points (Figures 1 and 2). They were classified as: 1) closed, if there was continuity in the inner retinal layers (retinal nerve fiber layer, ganglion cell layer complex, inner nuclear layer and outer plexiform layer) and complete flattening against the retinal pigment epithelium (RPE) layer of the outer retinal layers (outer nuclear layer, external limiting membrane, photoreceptor inner segment/outer segment junction layer, with or without disruption of these layers) (Figure 1A), 2) closed with FL, if there was continuity of the inner retinal layers with the presence of a hyporeflective space with or without disruption of outer retinal layers underlying the fovea (Figure1B and Figure 2B), and 3) open, if there was a persistent full thickness retinal defect with direct communication between the vitreous cavity and RPE (Figure 1C). The integrity of the external limiting membrane (ELM) was also evaluated. Quantitative measurements were performed manually with the caliper tool of the device. Preoperative macular hole size was defined by the minimum diameter and the maximum height of the macular holes, and postoperative foveolar lucency size was defined by the maximum diameter at different time points.

\section{Statistical Analysis}

Descriptive, frequency statistics, and the chi-squared test were used to assess qualitative variables. Normality of quantitative variables was examined using histograms. Independent Student t-test and one-way ANOVA was performed to assess quantitative variables between groups. Visual acuity measured in Snellen notation was converted to LogMAR equivalents for the 
purposes of statistical analysis. VA values recorded as counting fingers (CF), hand movement $(\mathrm{HM})$, and perception of light $(\mathrm{PL})$ were converted to 2.1, 2.4, and 2.7 LogMAR, respectively. ${ }^{13}$ Subgroup analysis was also performed for macular hole stage and type of gas tamponade employed. A $p$ value of less than 0.05 was considered statistically significant. All statistical analysis was performed using SPSS 15.0 software (SPSS Inc., Chicago, Illinois).

\section{RESULTS:}

\section{Baseline Characteristics}

A total of 142 eyes from 132 patients were included in the study. The preoperative characteristics and clinical features of patients included in the study are summarized in Table 1.

The mean age of patients was $68.6 \pm 8.6$ years (mean \pm SD)(median: 69.0 ) with a $3.25: 1$ female preponderance $(76.5 \%$ females, $101 / 132)$ and $7.5 \%$ of cases were bilateral (10/132). Macular holes were stage II in $28.9 \%$ of eyes, stage III in $57.7 \%$ and stage IV in $13.4 \%$. Regarding gas tamponade, $28.2 \%$ of eyes $(n=40)$ were treated with sulfahexafluoride (SF6), 43.0\% ( $n=61)$ with hexafluoroethane $(\mathrm{C} 2 \mathrm{~F} 6)$ and $28.9 \%(n=41)$ with perfluoropropane (C3F8). C3F8-treated eyes had significantly worse baseline VA $(1.08 \pm 0.45)$ than SF6 and C2F6-treated eyes $(0.84 \pm 0.30$ and $0.87 \pm 0.25$, respectively, $p$ $<0.01)$. No differences in macular hole stage $(p=0.95, p=0.36, p=0.31)$ or macular hole size (SF6 340.9, C2F6 356.4, C3F8 368.3 microns, $p=0.48$ ) were observed between gas tamponade groups. Eighty-three percent $(83.4 \%)$ 
of eyes were phakic and $16.6 \%$ were pseudophakic prior to surgery. PPV was combined with cataract surgery in $8.3 \%$ of operations.

\section{Clinical Features:}

\section{- Anatomical outcomes and prevalence of foveolar lucency:}

- Closure rate by spectral domain optical coherence tomography (SD-OCT):

At 1, 3, 6 and 12 months, closure of macular holes was achieved in $93.1 \%$ (94/101), 93.5\% (115/123), 94.6\% (122/129) and 95.5\% (127/133) of eyes respectively, with an overall final closure rate of $96.5 \%(137 / 142)$. One month after surgery, a closed macular hole with FL was observed in 33.7\% (34/101) of eyes, at 3 months this percentage was $7.3 \%$ (9/123), at 6 months $4.7 \%$ (6/129) and at 12 months was 3.0\% (4/133) (Table 2).

- Effect of gas tamponade, macular hole stage and size on closure pattern: One month after surgery, eyes treated with perfluoropropane (C3F8) showed a significantly lower percentage of closed holes with FL $(9.5 \%, 2 / 21)$ than eyes receiving hexafluoroethane $(\mathrm{C} 2 \mathrm{~F} 6)(40.9 \%, 18 / 44, \mathrm{p}=0.03)$, and a trend was observed when compared with sulfahexafluoride (SF6) (38.9\%, 14/36, p $=0.05)$ (Figure 3). Differences in FL rates were not associated with the stage of macular hole, $40.7 \%(11 / 27), 27.9 \%$ (17/61) and $46.2 \%(6 / 13)$ for stages II, III and IV, respectively. No association was observed between FL presence and preoperative macular hole diameter (329.4 vs. $355.8, p=0.46$ ) or height (404.8 vs. $377.3, p=0.15$ ). No correlation was found between FL size and preoperative macular hole diameter $(r=-0.037, \mathrm{p}=0.87)$ or height $(r=0.271$, $p=0.22)$. At three months, complete closure with flattening against the RPE was observed in $90.3 \%(28 / 31), 85.2 \%(46 / 54)$ and $84.2 \%(32 / 38)$, and at six 
months in $93.8 \%$ (30/32), 87.9\% (51/58) and 89.7\% (35/39) for C3F8, C2F6 and SF6 respectively. No significant differences between gases or macular hole stage were observed at the 3 or 6 -month time point.

- Visual outcomes and impact of foveolar lucency:

- Effect of foveolar lucency on VA change and mean VA: No significant differences were observed in mean VA improvement (change from baseline) between eyes with closed holes that exhibited FL and eyes with closed holes without FL at any of the studied time points. At 1 month after surgery, the mean VA improvement was $-0.14 \pm 0.19$ vs. $-0.11 \pm 0.23(p=0.48)$, respectively (Figure 4). At 3 months, the mean VA improvement was $-0.22 \pm$ 0.22 vs. $-0.33 \pm 0.36(p=0.37)$, and at 6 months it was $-0.34 \pm 0.28$ vs. -0.37 $\pm 0.28(p=0.74)$, respectively. No direct comparisons in mean actual VA were possible between these two subgroups since baseline VA before surgery was better for eyes with FL than those without FL at month $1(0.83 \pm 0.17$ vs. 0.91 $\pm 0.32, p=0.12)$. No differences in VA were observed between eyes with and without ELM disruption at month 1 (0.69 vs. 0.68 LogMAR, 20/97 vs. 20/95 Snellen, $p=0.93$ ) or month 3 (0.59 vs. 0.53 LogMAR, 20/77 vs. 20/67 Snellen $p=0.38)$. 


\section{DISCUSSION:}

This relatively large series of macular hole surgery showed a significant rate of FL during the course of macular hole closure with nearly $1 / 3$ of eyes exhibiting this feature 1 month after vitrectomy surgery. A new interesting finding in this study is that C3F8 gas appears to be associated with a lower rate of FL compared to shorter acting gases. In this series, the presence of FL was not found to have a significant impact on visual acuity at any time point.

The presence of FL on OCT after macular hole surgery has been previously reported. ${ }^{11,12,14,15}$ The origin of this hyporeflective space appears to be related to the healing process of macular holes. This begins with bridging of the inner retina mimicking a foveal detachment, which resolves or remains with time as an outer foveal defect. ${ }^{11,16,17}$ Little is known about which features predispose to this finding. A recent study has suggested that preoperative basal hole diameter may predict FL development, ${ }^{12}$ however, intraoperative OCT studies have demonstrated increased subretinal fluid and increased base area of fluid within a macular hole during ILM peeling, suggesting that mechanical traction during surgery could also be a causative factor. ${ }^{18,19}$ In our study, this feature was observed in $33.7 \%$ at 1 month, $7.3 \%$ at 3 months, $4.7 \%$ at 6 months and $3.0 \%$ at 12 months. These rates are lower than those reported by Grewal et al (FL rate of $71 \%, 32 / 45$, using both SF6 and C3F8) and Sano et al (FL rate of $43 \%, 12 / 28$, using SF6) ${ }^{17}$ at month 1 , and Christensen et al at month 3 (FL rate of $36.5 \%, 27 / 74$, using C3F8),${ }^{11}$ but 
slightly higher than the $26 \%$ reported by Mahmoud et al in another small series $(9 / 35)^{15}$. The differences in the percentage and timing of FL may be explained by our larger sample size (142 vs. $45,28,74,35$, respectively) and to some extent the surgical technique employed, as all eyes in our series underwent dye-assisted ILM peeling and in one of the studies a non-peeling group was included. ${ }^{11}$

This is the first study to investigate the influence of intraocular gas tamponade on the rate of FL after macular hole closure. A new finding in this study was the significantly lower rate of FL in closed holes where C3F8 (9.5\%) was employed compared to C2F6 (40.9\%) and SF6 (38.9\%), which was independent of the preoperative stage of macular hole. This observation, that long acting gas is associated with a lower rate of FL, could at least in part explain the differences in FL rates observed in previous studies that used different types of gas tamponade. ${ }^{11}, 17$ We hypothesize that the effect of intraocular gas tamponade on FL rate could be explained by the fact that a long acting gas as C3F8 provides longer periods of effective contact angle between the gas bubble and the retina compared to shorter acting gases as C2F6 and SF6. ${ }^{20}$ As intraocular pressure (IOP) in gas-filled eyes is directly related to aqueous dynamics and long acting gases are known to produce IOP elevations more frequently than shorter acting gases, ${ }^{21}$ it is possible that this may also influence the lower rate of FL observed in C3F8-treated eyes.

The visual impact of FL remains controversial, with several studies including the present series reporting no significant effect on visual outcomes at any time point. ${ }^{15,22}$ By contrast, the Copenhagen study found that the 
presence of FL was associated with significantly worse visual acuity at 3 months but no differences were evident at 12 months when the FL had resolved, suggesting a temporary effect on vision. ${ }^{11}$ In keeping with this previously published finding we found a trend for slightly delayed visual acuity recovery in closed holes with FL (Figure 4), however this did not reach statistical significance. It should be noted that in contrast to the Copenhagen study, our data were gathered from a routine clinical setting and visual acuity was determined as the best VA with habitual correction or pin-hole rather than best-corrected refracted acuity as in the context of a clinical trial.

The results of our study need to be interpreted with caution because of the risk of bias associated with non-randomization and its retrospective design. In addition, OCT scans were not routinely performed at all postoperative visits hence the data shown is a subset of the whole sample at every follow up time point. Moreover, we had data regarding the macular hole stage and size but not the symptom duration, which is known to be related to closure rate and final visual outcome..$^{23}$ The type of gas tamponade employed may have also affected the closure rates of macular holes but in our series each consultant favoured a different gas type for all grades of macular holes. The importance of strict face-down posturing after macular hole surgery, which was not used in our series, remains controversial but is not the primary determinant of macular hole closure. ${ }^{7,24}$

In conclusion, this study describes the anatomical patterns of OCT closure and prevalence of FL in a large series of patients undergoing macular hole surgery. This work suggests that the duration of gas tamponade 
influences the pattern of anatomical closure with lower rates of FL in eyes where long acting gases were used. Future use of new technologies such as adaptive optics, swept-source OCT or ultrahigh resolution OCT may allow greater predictive value on the effects of FL resolution on the final anatomical recovery of retinal layers. 


\section{REFERENCES:}

1. Gass JD. Idiopathic senile macular hole. Its early stages and pathogenesis. Arch Ophthalmol 1988; 106:629-639.

2. McCannel CA et al. Population-based incidence of macular holes. Ophthalmology 2009; 116:1366-1369.

3. Jackson TL et al. United Kingdom National Ophthalmology Database Study of Vitreoretinal Surgery: report 1; case mix, complications, and cataract. Eye (Lond) 2013; 27:644-651.

4. Kim JW et al. Prospective randomized trial of vitrectomy or observation for stage 2 macular holes. Vitrectomy for Macular Hole Study Group. Am J Ophthalmol 1996; 121:605-614.

5. Freeman WR et al. Vitrectomy for the treatment of full-thickness stage 3 or 4 macular holes. Results of a multicentered randomized clinical trial. The Vitrectomy for Treatment of Macular Hole Study Group. Arch Ophthalmol $1997 ; 115: 11-21$.

6. Scott IU et al. Long-term anatomic and visual acuity outcomes after initial anatomic success with macular hole surgery. Am J Ophthalmol 2003; 135:633-640.

7. Essex RW et al. The Effect of Postoperative Face-Down Positioning and of Long- versus Short-Acting Gas in Macular Hole Surgery: Results of a Registry-Based Study. Ophthalmology 2016; 123:1129-1136. 
8. Lois $\mathrm{N}$ et al. Internal limiting membrane peeling versus no peeling for idiopathic full-thickness macular hole: a pragmatic randomized controlled trial. Invest Ophthalmol Vis Sci 2011; 52:1586-1592.

9. Michalewska Z et al. Correlation between foveal structure and visual outcome following macular hole surgery: a spectral optical coherence tomography study. Graefes Arch Clin Exp Ophthalmol 2008; 246:823-830.

10. Wakabayashi T et al. Foveal microstructure and visual acuity in surgically closed macular holes: spectral-domain optical coherence tomographic analysis. Ophthalmology 2010; $117: 1815-1824$.

11. Christensen UC et al. Prognostic significance of delayed structural recovery after macular hole surgery. Ophthalmology 2009; 116:2430-2436.

12. Grewal DS, Reddy V and Mahmoud TH. Assessment of Foveal Microstructure and Foveal Lucencies Using Optical Coherence Tomography Radial Scans Following Macular Hole Surgery. Am J Ophthalmol 2015; 160:990-999 e991.

13. Sparrow JM et al. The Cataract National Dataset electronic multicentre audit of 55,567 operations: risk indicators for monocular visual acuity outcomes. Eye (Lond) 2012; 26:821-826.

14. Moshfeghi AA et al. Persistent outer retinal defect after successful macular hole repair. Am J Ophthalmol 2005; 139:183-184.

15. Mahmoud TH and McCuen BW, 2nd. Natural history of foveolar lucencies observed by optical coherence tomography after macular hole surgery. Retina $2007 ; 27: 95-100$. 
16. Hangai $\mathrm{M}$ et al. Three-dimensional imaging of macular holes with highspeed optical coherence tomography. Ophthalmology 2007; 114:763-773.

17. Sano M et al. Restored photoreceptor outer segment and visual recovery after macular hole closure. Am J Ophthalmol 2009; 147:313-318 e311.

18. Ray R et al. Intraoperative microscope-mounted spectral domain optical coherence tomography for evaluation of retinal anatomy during macular surgery. Ophthalmology 2011; 118:2212-2217.

19. Ehlers JP et al. Intrasurgical dynamics of macular hole surgery: an assessment of surgery-induced ultrastructural alterations with intraoperative optical coherence tomography. Retina 2014; 34:213-221.

20. Eames I et al. A theoretical model for predicting interfacial relationships of retinal tamponades. Invest Ophthalmol Vis Sci 2010; 51:2243-2247.

21. Hutter J, Luu H and Schroeder L. A biological model of tamponade gases following pneumatic retinopexy. Curr Eye Res 2002; 25:197-206.

22. Shimozono M et al. Restoration of the photoreceptor outer segment and visual outcomes after macular hole closure: spectral-domain optical coherence tomography analysis. Graefes Arch Clin Exp Ophthalmol 2011; 249:1469-1476.

23. Ip MS et al. Anatomical outcomes of surgery for idiopathic macular hole as determined by optical coherence tomography. Arch Ophthalmol 2002; 120:29-35.

24. Dhawahir-Scala FE et al. To posture or not to posture after macular hole surgery. Retina 2008; 28:60-65. 


\section{Figure legends:}

\section{Figure 1.}

Macular hole status by spectral domain optical coherence tomography (SDOCT). Macular hole status preoperatively (left) and postoperatively (right) one month after surgery by SD-OCT. A. Closed hole with complete flattening of outer retinal layers against the RPE. B. Closed hole with foveolar lucency (subfoveal hyporeflective space with continuity of inner retinal layers). C. Open hole after unsuccessful macular hole surgery.

\section{Figure 2.}

Macular hole closure process by spectral domain optical coherence tomography (SD-OCT). A. Preoperative status with full thickness open macular hole. B. Closed macular hole with foveolar lucency 1 month after surgery. C. Closed macular hole with complete flattening of outer retinal layers 3 months after surgery.

Figure 3.

Anatomical outcomes and foveolar lucency rates by spectral domain optical coherence tomography (SD-OCT). A-B: Subanalysis per gas tamponade; and C-D: subanalysis per stage of macular holes. A, C: Closure rates at $1^{\text {st }}$ month. B, D: Closure rates at $3^{\text {rd }}$ month.

\section{Figure 4.}

Visual Acuity (VA) outcomes in closed holes with and without foveolar lucency. A. Mean VA at different time points. No significant differences were 
observed at any of the postoperative time points. B. Mean VA change from baseline at different time points. 
A

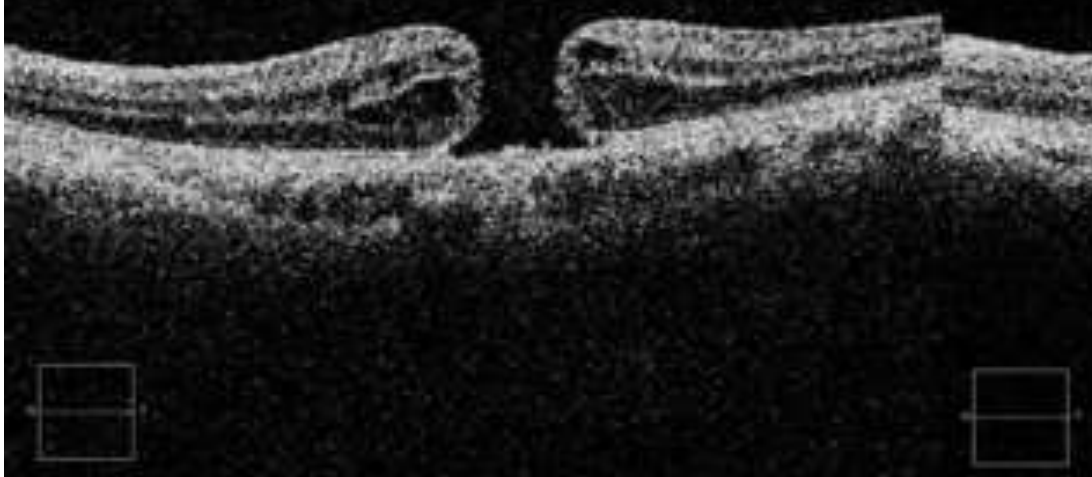

B

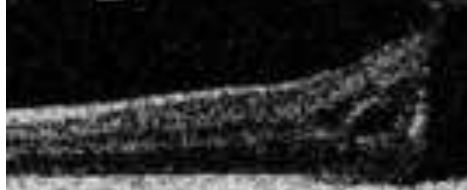

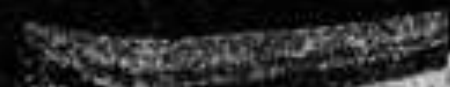

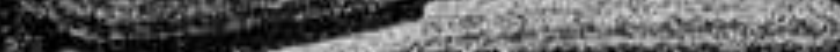

sociot
$\square$
$\square$

ses

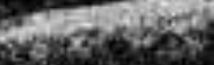

C

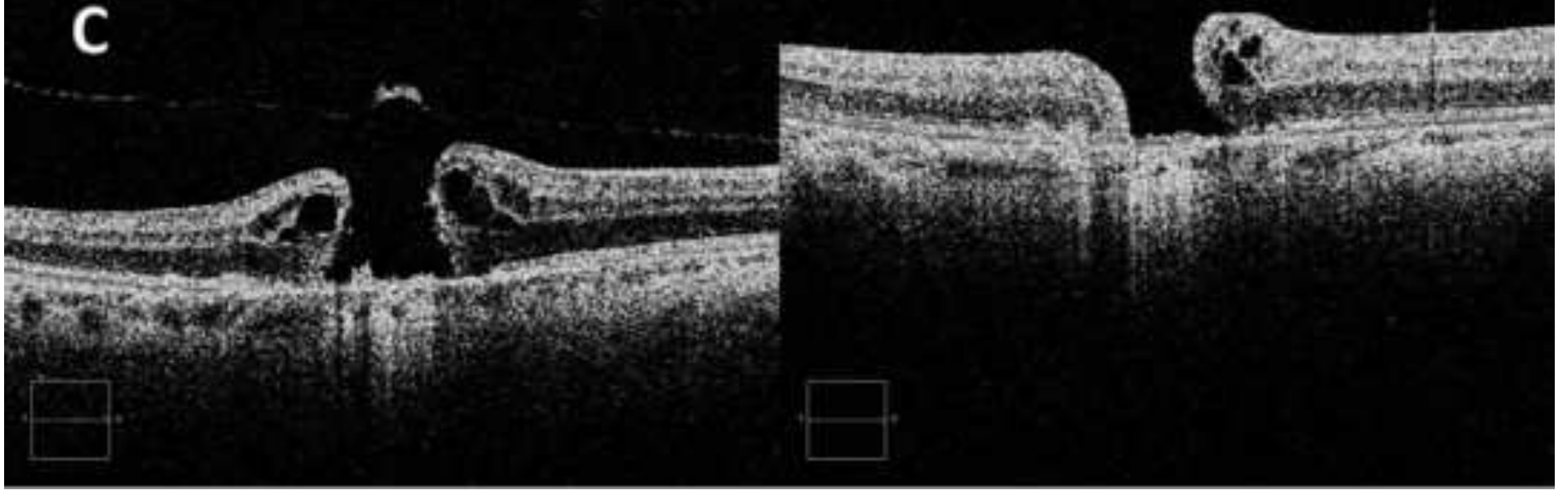




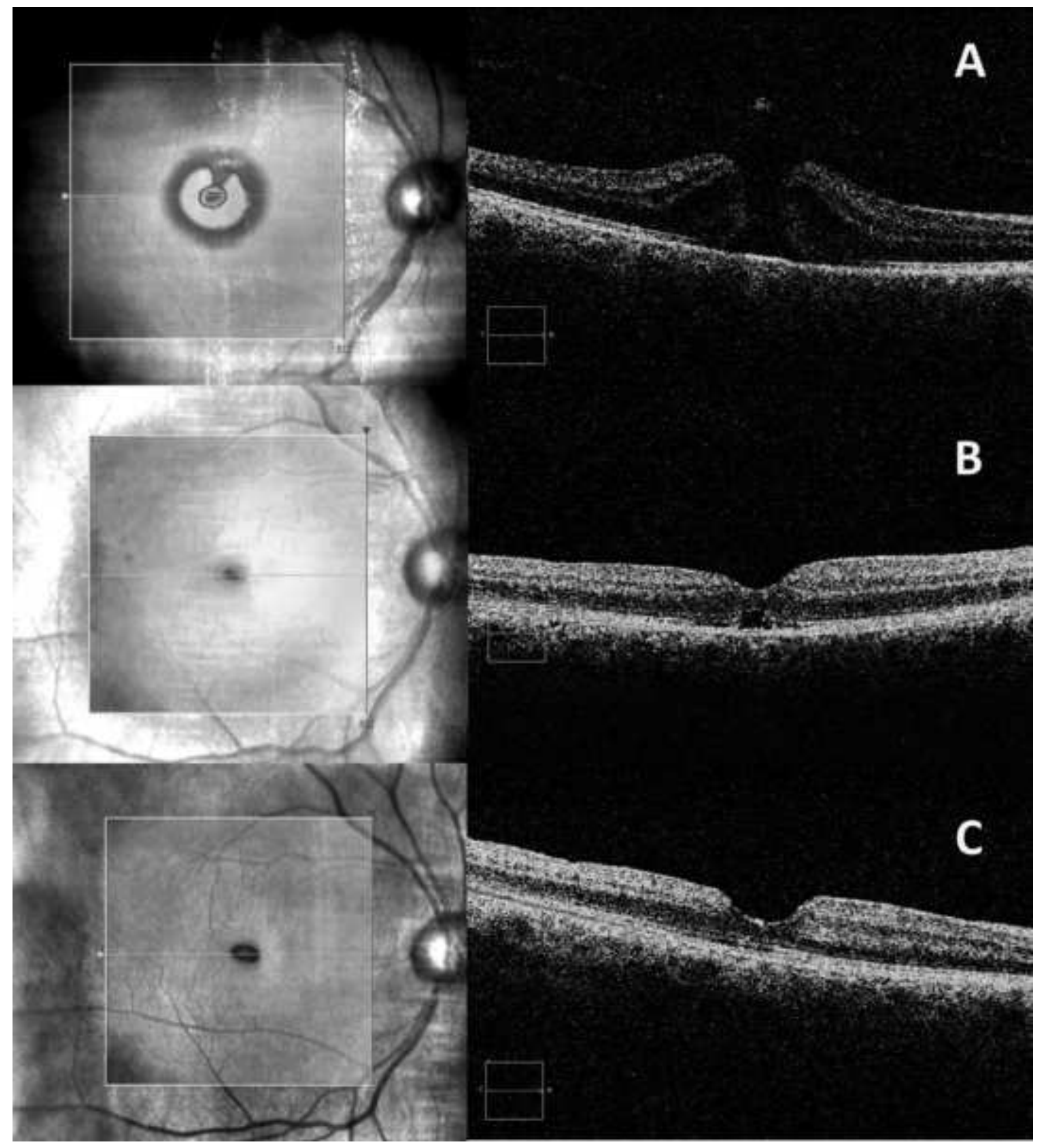




\section{A 1 month - \% Anatomical Closure by OCT \& Gas}

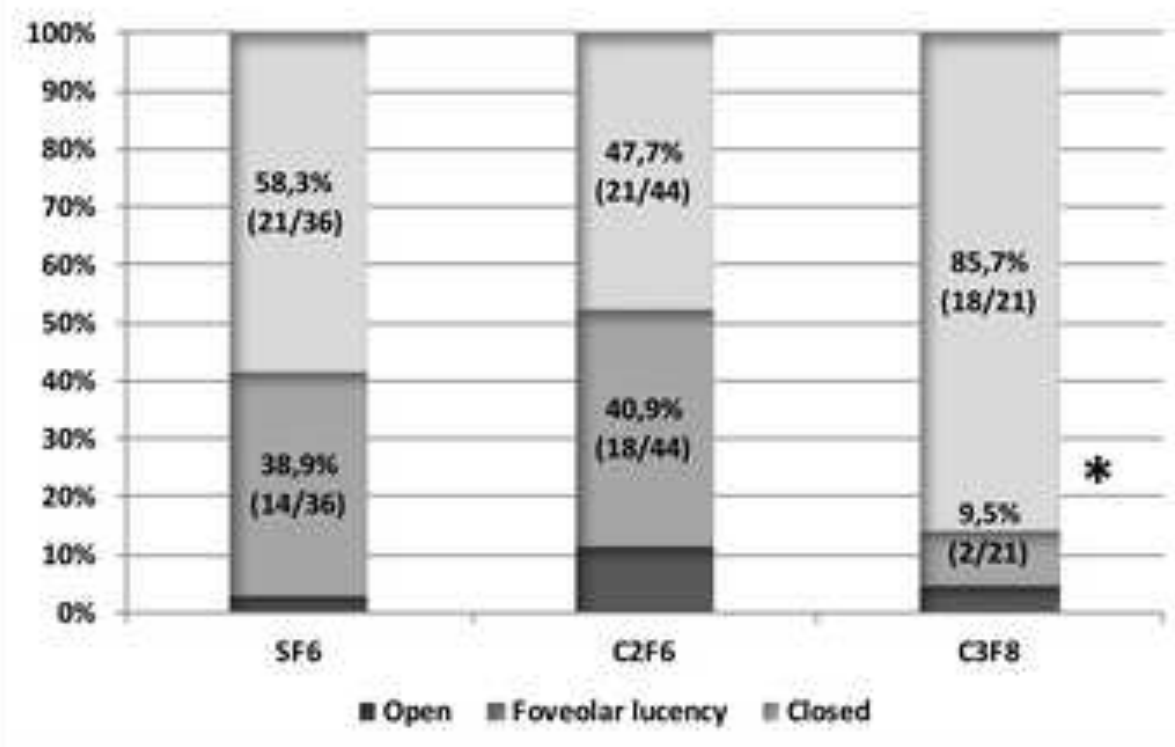

C 1 month-\% Anatomical Closure by OCT \& Stage

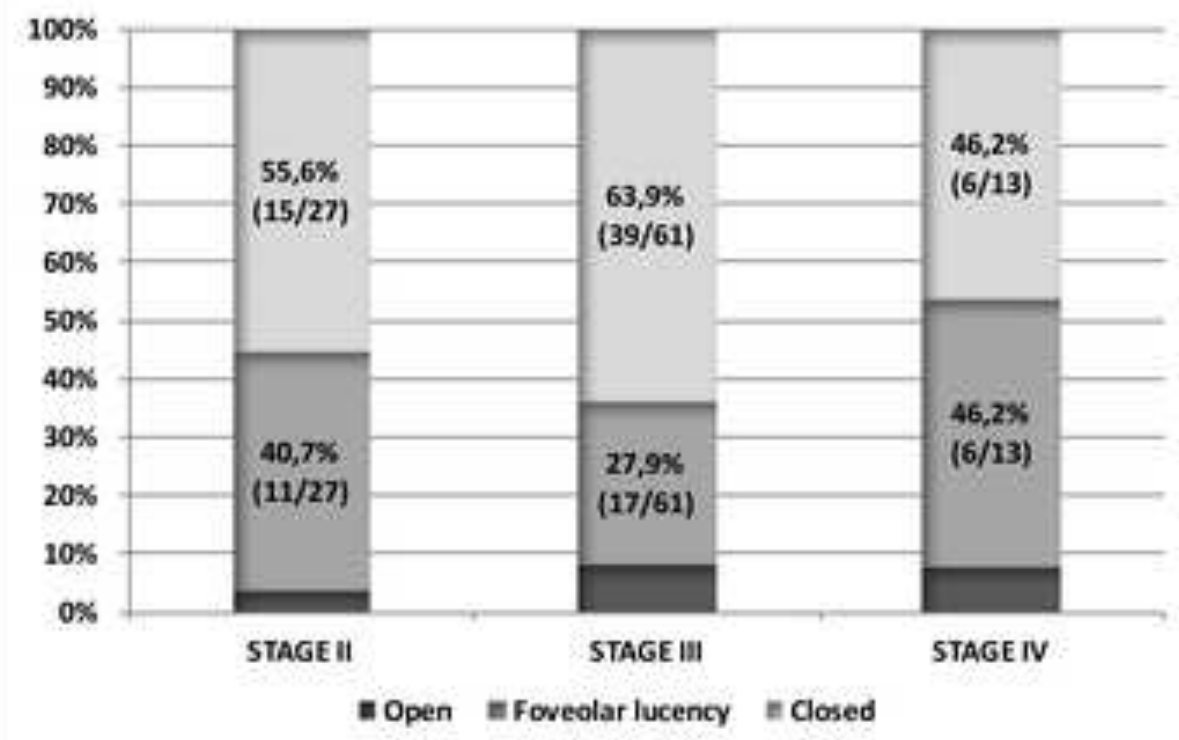

\section{B 3 month - \% Anatomical Closure by OCT \& Gas}

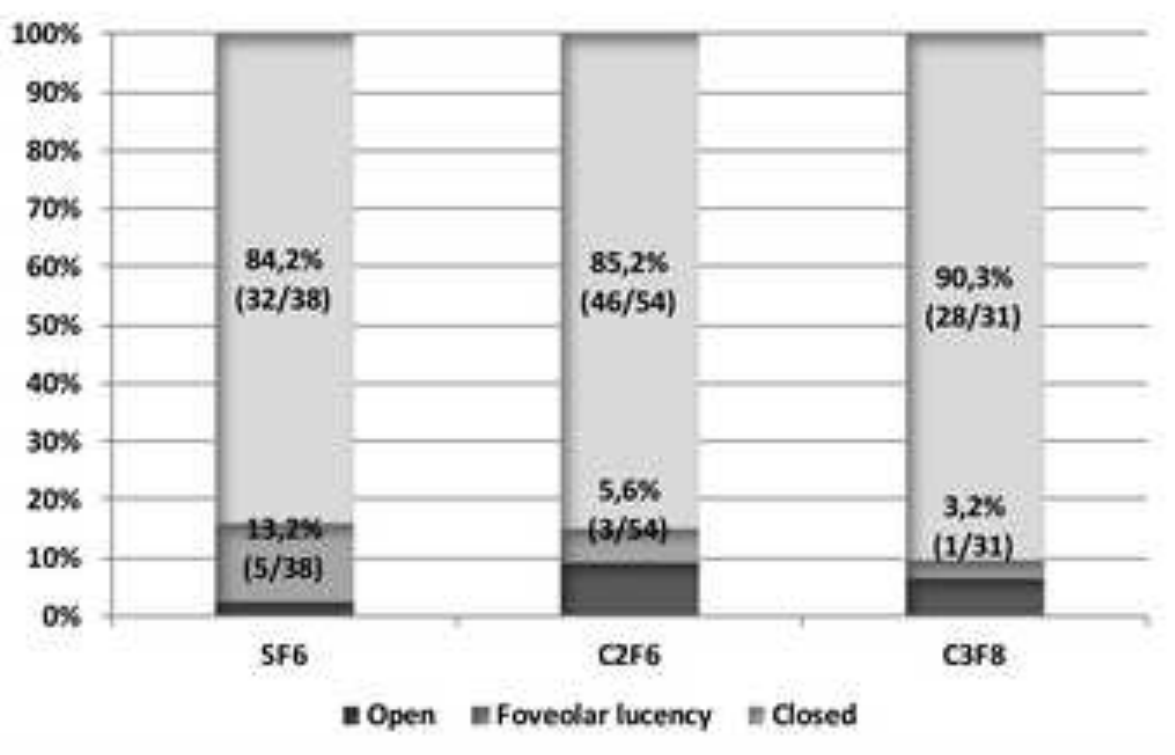

D 3 month - \% Anatomical Closure by OCT \& Stage

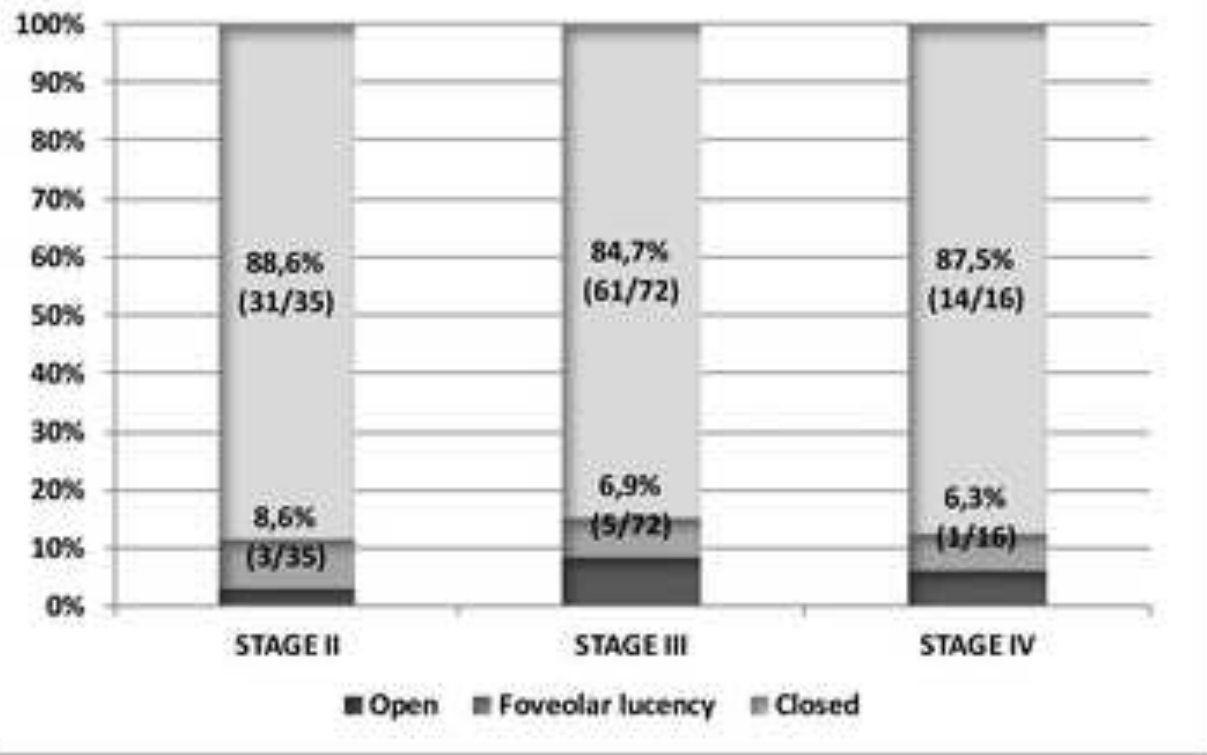




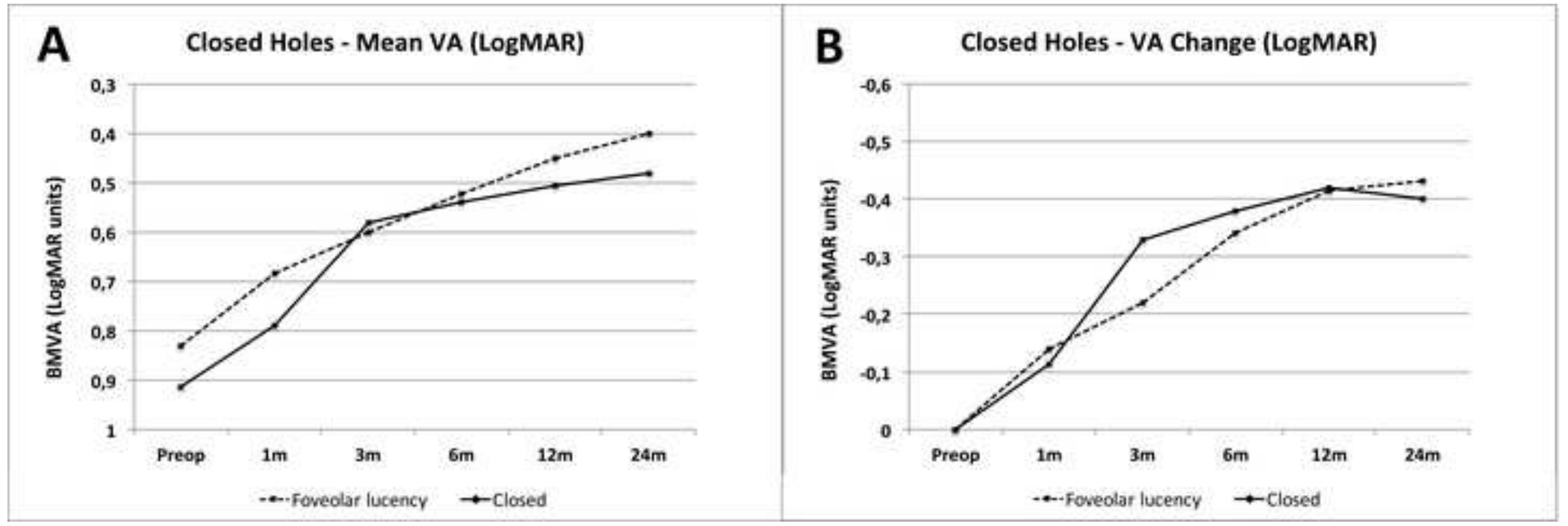


Table 1. Demographics and clinical features of patients included in the study. Subgroup analysis by gas tamponade.

\begin{tabular}{|c|c|c|c|c|c|c|}
\hline & & TOTAL & SF6 & $\mathrm{C} 2 \mathrm{~F} 6$ & C3F8 & $p$ value \\
\hline $\mathbf{n}$ & & $142(100 \%)$ & $40(28.2 \%)$ & $61(43.0 \%)$ & $41(28.8 \%)$ & \\
\hline Age (years) & Mean+/-SD & $68.6 \pm 8.6$ & $68.7 \pm 7.9$ & $68.9 \pm 7.3$ & $67.9 \pm 10.9$ & 0.84 \\
\hline \multirow{2}{*}{ Gender $^{\mathrm{A}}$} & Female & $77.5 \%(110 / 142)$ & $70.0 \%(28 / 40)$ & $85.2 \%(52 / 61)$ & $73.2 \%(30 / 41)$ & \multirow{2}{*}{0.14} \\
\hline & Male & $22.5 \%(32 / 142)$ & $30.0 \%(12 / 40)$ & $14.8 \%(9 / 61)$ & $26.8 \%(11 / 41)$ & \\
\hline \multirow{3}{*}{ Macular hole Stage } & Stage II & $28.9 \%(41 / 142)$ & $32.5 \%(13 / 40)$ & $26.2 \%(16 / 61)$ & $29.3 \%(12 / 41)$ & 0.95 \\
\hline & Stage III & $57.7 \%(82 / 142)$ & $60.0 \%(24 / 40)$ & $55.7 \%(34 / 61)$ & $58.5 \%(24 / 41)$ & 0.36 \\
\hline & Stage IV & $13.4 \%(19 / 142)$ & $7.5 \%(3 / 40)$ & $18.0 \%(11 / 61)$ & $12.2 \%(5 / 41)$ & 0.31 \\
\hline \multirow[t]{2}{*}{ Macular hole size } & Diameter & 340.9 & 319.4 & 356.4 & 368.3 & 0.48 \\
\hline & $\begin{array}{l}\text { Maximum } \\
\text { height }\end{array}$ & 392.6 & 400.9 & 385.6 & 387.0 & 0.66 \\
\hline $\begin{array}{l}\text { Baseline Visual Acuity } \\
\text { (LogMAR) } \\
\text { (Snellen) }\end{array}$ & Mean+/-SD & $\begin{array}{c}0.92 \pm 0.30 \\
(20 / 166)\end{array}$ & $\begin{array}{c}0.84 \pm 0.30 \\
(20 / 138)\end{array}$ & $\begin{array}{c}0.87 \pm 0.25 \\
(20 / 148)\end{array}$ & $\begin{array}{c}1.08 \pm 0.45 \\
(20 / 240)\end{array}$ & $<0.01$ \\
\hline \multirow{2}{*}{$\begin{array}{l}\text { Phakic Status } \\
(92.9 \%)^{\mathrm{B}}\end{array}$} & Yes & $77.4 \%(110 / 142)$ & $80.0 \%(32 / 40)$ & $75.4 \%(46 / 61)$ & $78.0 \%(32 / 41)$ & \multirow{2}{*}{0.96} \\
\hline & No & $15.4 \%(22 / 142)$ & $17.5 \%(7 / 40)$ & $14.7 \%(9 / 61)$ & $14.6 \%(6 / 41)$ & \\
\hline
\end{tabular}

Table 1. Demographics and clinical features of patients included in the study. Subgroup analysis by gas tamponade. SF6 = sulfahexafluoride, C2F6 = hexafluoroethane, C3F8 = perfluoropropane, SD = standard deviation. Significance level $=p<0.05$ (chisquare test). ( ${ }^{A}$ gender per eye: bilateral cases considered individually, ${ }^{B}$ percentage data available). 

Table 2. Closure rate as per spectral domain optical coherence tomography (SD-OCT) and gas tamponade.

\begin{tabular}{|c|c|c|c|c|c|c|}
\hline \multicolumn{2}{|c|}{ Closure rate (SD-OCT) - gas tamponade } & $1 \mathrm{~m}$ & $3 m$ & $6 m$ & $12 m$ & Overall \\
\hline \multirow{3}{*}{ SF6 } & Closed & $58.3 \%(21 / 36)$ & $84.2 \%(32 / 38)$ & $89.7 \%(35 / 39)$ & $94.9 \%(37 / 39)$ & $97.5 \%(39 / 40)$ \\
\hline & Closed-FL & $38.9 \%(14 / 36)$ & $13.2 \%(5 / 38)$ & $7.7 \%(3 / 39)$ & $5.1 \%(2 / 39)$ & $2.5 \%(1 / 40)$ \\
\hline & Open & $2.8 \%(1 / 36)$ & $2.6 \%(1 / 38)$ & $2.6 \%(1 / 39)$ & $0.0 \%(0 / 39)$ & $0.0 \%(0 / 40)$ \\
\hline \multirow[t]{2}{*}{$\mathrm{C} 2 \mathrm{~F} 6$} & Closed-FL & $40.9 \%(18 / 44)$ & $5.6 \%(3 / 54)$ & $3.4 \%(2 / 58)$ & $1.6 \%(1 / 61)$ & $1.6 \%(1 / 61)$ \\
\hline & Open & $11.4 \%(5 / 44)$ & $9.3 \%(5 / 54)$ & $8.6 \%(5 / 58)$ & $8.2 \%(5 / 61)$ & $6.6 \%(4 / 61)$ \\
\hline \multirow[b]{2}{*}{ C3F8 } & Closed & $85.7 \%(18 / 21)$ & $90.3 \%(28 / 31)$ & $93.8 \%(30 / 32)$ & $93.9 \%(31 / 33)$ & $95.1 \%(39 / 41)$ \\
\hline & Closed-FL & $9.5 \%(2 / 21)$ & $3.2 \%(1 / 31)$ & $3.1 \%(1 / 32)$ & $3.0 \%(1 / 33)$ & $2.4 \%(1 / 41)$ \\
\hline \multirow{4}{*}{ TOTAL } & Closed & $59.4 \%(60 / 101)$ & $86.2 \%(106 / 123)$ & $89.9 \%(116 / 129)$ & $92.5 \%(123 / 133)$ & $94.4 \%(134 / 142)$ \\
\hline & Closed-FL & $33.7 \%(34 / 101)$ & $7.3 \%(9 / 123)$ & $4.7 \%(6 / 129)$ & $3.0 \%(4 / 133)$ & $2.1 \%(3 / 142)$ \\
\hline & Open & $6.9 \%(7 / 101)$ & $6.5 \%(8 / 123)$ & $5.4 \%(7 / 129)$ & $4.5 \%(6 / 133)$ & $3.5 \%(5 / 142)$ \\
\hline & $\%$ (n/Total) & $71.1 \%(101 / 142)$ & $86.6 \%(123 / 142)$ & $90.8 \%(129 / 142)$ & $93.6 \%(133 / 142)$ & $100 \%(142 / 142)$ \\
\hline
\end{tabular}

Table 2. Percentage of closed holes with and without foveal lucency at each time point. Closed-FL = Closed holes with Foveal Lucency, SF6 = sulfahexafluoride, C2F6 = hexafluoroethane, C3F8 = perfluoropropane. $(\%$ of total eyes with data at that time point (n/total). 